\title{
Combining text/image in WikipediaMM task 2009
}

\author{
Christophe Moulin, Cécile Barat, Cédric Lemaître, Mathias Géry, Christophe \\ Ducottet, Christine Largeron \\ Université de Lyon, F-69003, Lyon, France \\ Université de Saint-Étienne, F-42000, Saint-Étienne, France \\ CNRS UMR5516, Laboratoire Hubert Curien \\ \{christophe.moulin, cecile.barat, cedric.lemaitre, mathias.gery, \\ ducottet, largeron\}@univ-st-etienne.fr
}

\begin{abstract}
This paper reports our multimedia information retrieval experiments carried out for the ImageCLEF track 2009. In 2008, we proposed a multimedia document model defined as a vector of textual and visual terms weighted using a tf.idf approch [5]. For our second participation, our goal was to improve this previous model in the following ways: 1) use of additional information for the textual part (legend and image bounding text extracted from the original documents, 2) use of different image detectors and descriptors, 3) new text / image combination approach. Results allow to evaluate the benefits of these different improvements.
\end{abstract}

\section{Introduction}

ImageCLEFwiki is a multimedia collection where each document is composed of text and one image. User needs are represented by queries ("topics"), which are also multimedia. Therefore, a multimedia document model is necessary to handle such a collection. In 2008, we proposed a first model that combines text and image information for multimedia retrieval [5]. This year, we improve our model adding textual information and using different detectors and descriptors for the visual information. Moreover we use a linear combination to merge our textual and visual results. After presenting our model, we will explain the submitted runs and the obtained results. We will finish by introducing our future work.

\section{Visual and textual document model}

The document model we defined for ImageCLEF 2008 lets us rank documents depending on the query using different methods. Firstly, we explain the key features of our approach to rank documents according to a query using only textual information. Secondly, we describe how we extend the method to handle the visual information. Finally, we present our method for combining textual and visual results. 


\subsection{Textual representation model}

As in the vector space model introducted by Salton et al. [7], we represent a document $d_{i}$ as a vector of weights $w_{i, j}$. Each $w_{i, j}$ corresponds to the importance of the term $t_{j}$ in the document $d_{i}$ computed by multiplying $t f_{i, j}$ and $i d f_{j}$, where $t f_{i, j}$ is the term frequency that characterizes the frequency of the term $t_{j}$ in the document $d_{i}$. The $i d f_{j}$ is the inverse document frequency that quantifies the importance of the term $t_{j}$ over the corpus of documents. $w_{i, j}$ is high when the term $t_{j}$ is frequent in the document $d_{i}$ but rare in the others. We use $t f_{i, j}$ and $i d f_{j}$ defined in the Okapi formula by Robertson et al [6] by :

$$
t f_{i, j}=\frac{k_{1} n_{i, j}}{n_{i, j}+k_{2}\left(1-b+b \frac{\left|d_{i}\right|}{d_{a v g}}\right)}
$$

where $n_{i, j}$ is the occurrence of the term $t_{j}$ in the document $d_{i},\left|d_{i}\right|$ the size of the document $d_{i}$ and $d_{\text {avg }}$ the average size of all documents in the corpus and $k_{1}, k_{2}$ and $b$ are three constants.

$$
i d f_{j}=\log \frac{|D|-\left|\left\{d_{i} \mid u_{j} \in d_{i}\right\}\right|+0.5}{\left|\left\{d_{i} \mid t_{j} \in d_{i}\right\}\right|+0.5}
$$

where $|D|$ is the size of the corpus and $\left|\left\{d_{i} \mid t_{j} \in d_{i}\right\}\right|$ the number of documents where the term $t_{j}$ occurs at least one time.

If we consider a query $q_{k}$ as a short document, we can represent it as a vector of weights. A score is then computed between the query $q_{k}$ and a document $d_{i}$ as shown in table 1. The main difference between score $e^{1}$ and $s c o r e^{2}$ is the representation of the query. In the first score, the weight of the query is defined by its $t f$ only while in the second score the weight is equal to $t f . i d f$. Note that for $t f_{k, j}, b=0$ because $\left|d_{k}\right|$ and $\left|d_{a v g}\right|$ are not defined for a query.

\begin{tabular}{|c|l|l|}
\hline Scoring & $\begin{array}{l}\text { Parameters } \\
t f_{i, j}\end{array}$ & $\begin{array}{l}\text { Parameters } \\
t f_{k, j}\end{array}$ \\
\hline score $^{1}\left(q_{k}, d_{i}\right)=$ & $k_{1}=2.2$ & $k_{1}=8$ \\
$\sum_{t_{j} \in q_{k}} t f_{i, j} i d f_{j} t f_{k, j}$ & $k_{2}=1.2$ & $k_{2}=7$ \\
& $b=0.75$ & $b=0$ \\
\hline score $^{2}\left(q_{k}, d_{i}\right)=$ & $k_{1}=1$ & $k_{1}=1$ \\
$\sum_{t_{j} \in q_{k}} t f_{i, j} i d f_{j} t f_{k, j} i d f_{j}$ & $k_{2}=1$ & $k_{2}=1$ \\
& $b=0.5$ & $b=0$ \\
\hline
\end{tabular}

Table 1. Scoring equations and their default parameters[8].

Different sources of text are available. The legend provided with images is often very short and sometimes useless: for example, when the text deals with the copyright of the image or when it gives details about the user who uploaded the image. In order to gain information, we aim at using the original text extracted 
from the wikipedia documents in which images appear. We consider a text fragment aroung the image. The size of the window is tuned using wikipediaMM 2008 collection as a training collection. We add this text to the legend of the image and we index both the added text and the original legend. The indexing is performed with the Lemur software[8].

\subsection{Visual representation model}

In order to combine the visual information with the textual one, we also represent images as a vector of weights. Provided we are able to extract visual words from images, it is possible to use the $t f . i d f$ formula in the same way as in the textual model. It is therefore necessary to create a visual vocabulary $V=\left\{v_{1}, \ldots, v_{j}, \ldots, v_{|V|}\right\}$ as in [2]. For that purpose, we use 3 different descriptors. The first one (meanstd) is the same as in [5]. Each image is partitioned into $16 \times 16$ cells. Each cell is described by a six dimensional vector which corresponds to the mean and the standard deviation of $\frac{R}{R+G+B}, \frac{G}{R+G+B}$ and $\frac{R+G+B}{3 * 255}$ where $R, G$ and $B$ are the red, green and blue components of the cell. The second (named sift $t_{1}$ ) and third (named sift $t_{2}$ ) descriptors are based on the well

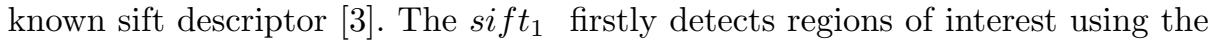
MSER method as in [4] while the sift $t_{2}$ one uses a regular partitioning as in the meanstd descriptor.

For each of our 3 descriptors, we apply a $k$-means algorithm [1] to obtain a vocabulary of 10'000 visual terms. Each visual term represents a cluster of feature vectors.

Then, each image can be represented using a vector of visual terms. Local features are first calculated using one of the 3 descriptors. Then visual terms are determined by seeking, for each feature, the closest visual term (according to the euclidian distance) in the corresponding visual vocabulary.

In the same way as for textual words, the weight of each visual term is computed using a $t f . i d f$ approach.

\subsection{Combination}

In order to combine textual and visual results we use two different methods. The first one is a simple intersection between the results obtained with the textual query and with the visual one. The second one corresponds to a linear combination between the textual and the visual scores.

$$
\operatorname{score}\left(q_{k}, d_{i}\right)=\operatorname{\alpha score}_{V}\left(q_{k}, d_{i}\right)+(1-\alpha) \operatorname{score}_{T}\left(q_{k}, d_{i}\right)
$$

The $\alpha$ parameter lets us add more or less visual information. We calculate its optimal value using the queries from the ImageCLEFwiki 2008 track as a training set. 


\section{$3 \quad$ Experiments}

Using the model described in the previous section, we present runs submitted to ImageCLEFwiki and the results we obtained.

\subsection{Submitted runs}

\begin{tabular}{|c|c|c|c|c|c|}
\hline run_id & run & score & text & image & combination \\
\hline$\overline{L a H C \_1}$ & LaHC_TXT_okapi & score $^{1}$ & legend & - & - \\
\hline $\mathrm{LaHC} \_2$ & LaHC_TXT_tfidf & score $^{2}$ & legend & - & - \\
\hline$L a H C \_3$ & run_inter_TXT_IMG_Meanstd & score $^{2}$ & legend & meanstd & intersection \\
\hline $\mathrm{LaHC}_{-} 4$ & run_inter_TXT_IMG_Sift & score $^{2}$ & legend & sift $_{1}$ & intersection \\
\hline$L a H C \_5$ & run_TXTIMG_Meanstd_0.015 & score $^{2}$ & legend & meanstd & $\alpha=0.015$ \\
\hline$L a H C \_6$ & run_TXTIMG_Meanstd_0.025 & score $^{2}$ & legend & meanstd & $\alpha=0.025$ \\
\hline $\mathrm{LaHC}_{-} 7$ & run_TXTIMG_Sift_0.012 & score $^{2}$ & legend & sift $_{1}$ & $\alpha=0.012$ \\
\hline LaHC_8 & run_inter_TXT_IMG_Siftdense & score $^{2}$ & legend & sift $_{2}$ & intersection \\
\hline$L a H C \_9$ & run_TXT_100_3_1_5 & score $^{2}$ & 100 char & legend & - \\
\hline$L a H C \_10$ & run_TXT_50_3_1_5 & score $^{2}$ & 50 char & legend & - \\
\hline$L a H C \_11$ & run_TXTIMG_100_3_1_5_meanstd & score $^{2}$ & 100 char & meanstd & $\alpha=0.025$ \\
\hline LaHC_12 & run_TXTIMG_50_3_1_5_meanstd & score $^{2}$ & 50 char & meanstd & $\alpha=0.025$ \\
\hline$L a H C \_13$ & run_TXTIMG_Siftdense_0.084 & score $^{2}$ & legend & sift $_{2}$ & $\alpha=0.084$ \\
\hline
\end{tabular}

- meanstd: regular partitioning + color descriptor

- sift $t_{1}$ MSER detector + sift descriptor

- sift $t_{2}$ : regular partitioning + sift descriptor

- legend: text of the image document

- $n$ char: size ( $n$ characters) of the text window around the image in the original wikipedia documents

Table 2. Presentation of the runs

All the runs are entirely automatic and are summarized on table 2 . We define a baseline, $L a H C_{\_} 1$, that corresponds to a pure text model. It uses only textual terms for the query and scoring of documents. We calculate the score $e^{1}$ for each image using terms of the textual content. The image name or bounding characters are not considered. We do not use neither feedback nor query expansion. Since score $e^{1}$ is applied, the query terms are weighted with their frequency $t f$.

Using only the text, we perform 3 other runs: the $L a H C_{-} 2$ is the same as the baseline except that the query is represented by its $t f . i d f$ rather than its $t f$. The $L a H C \_9$ and $L a H C \_10$ are two other text only runs that make use of the bounding text around the image in the original wikipedia document. The LaHC_9 adds 100 characters before and after the image while the $L a H C \_10$ adds 50 characters. 
All other runs exploit both the textual and the visual information of documents. The $L a H C \_3, L a H C \_4$ and $L a H C \_8$ are obtained after an intersection of the text only query results $\left(\mathrm{LaHC}_{2} 2\right)$ and the image query using the meanstd, the sift $t_{1}$ and the sift $t_{2}$ descriptors. The other runs are obtained from a linear combination of the textual and the visual scores. $L a H C \_5, L a H C \_6$, $L a H C \_7$ and $L a H C \_13$ use the textual scores of $L a H C \_2$ and the visual scores of a visual descriptor (meanstd, sift $t_{1}$ and sift $t_{2}$ ). LaHC_11 and LaHC_12 combine the textual scores of $L a H C \_9$ and $L a H C \_10$ with the visual scores of the meanstd descriptor.

\section{Results}

All the obtained results are summarized in Table 3. On the whole results, our team ranks 2 nd on 8 participants ${ }^{1}$. As we can see, the best results are obtained when we combine the image bounding text and the meanstd descriptor. We could have obtained better results if we had combined the image bounding text and the sift $t_{2}$ descriptor. Indeed, comparing results of text-image runs $L a H C \_6$, LaHC_7 and $L a H C_{-} \_13$, we can notice that the best visual descriptor is sift ${ }_{2}$, followed by meanstd and sift $t_{1}$. The last three results obtained after an intersection are the worst results, but if we compare them in term of precision, they are the best ones. Indeed, one document over six is relevant. As a rule, combining the textual information with the visual one always improves the results and return more relevant documents which is very encouraging.

\begin{tabular}{|c|c|c|c|c|}
\hline rank & run & map & num_ret & num_rel_ret \\
\hline \hline 5 & run_TXTIMG_100_3_1_5_meanstd & 0.2178 & 44993 & 1213 \\
\hline 6 & run_TXTIMG_50_3_1_5_meanstd & 0.2148 & 44993 & 1218 \\
\hline 14 & run_TXTIMG_Siftdense_0.084 & 0.1903 & 44993 & 1212 \\
\hline 15 & run_TXT_100_3_1_5 & 0.1890 & 38004 & 1205 \\
\hline 16 & run_TXT_50_3_1_5 & 0.1880 & 37041 & 1198 \\
\hline 20 & run_TXTIMG_Meanstd_0.025 & 0.1845 & 44993 & 1208 \\
\hline 21 & run_TXTIMG_Sift_0.012 & 0.1807 & 44995 & 1200 \\
\hline 24 & run_TXTIMG_Meanstd_0.015 & 0.1792 & 44993 & 1213 \\
\hline 33 & LaHC_TXT_tfidf & 0.1667 & 35611 & 1192 \\
\hline 44 & LaHC_TXT_okapi & 0.1432 & 35611 & 1164 \\
\hline 52 & run_inter_TXT_IMG_Siftdense & 0.0365 & 619 & 142 \\
\hline 53 & run_inter_TXT_IMG_Meanstd & 0.0338 & 574 & 76 \\
\hline 54 & run_inter_TXT_IMG_Sift & 0.0321 & 637 & 120 \\
\hline
\end{tabular}

Table 3. Presentation of the results

\footnotetext{
${ }^{1}$ http://imageclef .org/2009/wikiMM-results
} 


\section{Conclusion}

In this article we proposed improvements to our multimedia model we introduced in [5]. The first one was to use image bounding text extracted from the original documents, the second was to use sift based image descriptors for the visual part and the third one was to add a text/image combination approach. A series of thirteen runs was submitted using the ImageCLEFwiki 2009 collection. The first analysis of the results allowed to make the three following remarks. It's better to use the image bounding text than the legend only. The sift descriptor is better than our previous color descriptor provided it is calculated on a regular partitioning. The text-image combination is a winning strategy which can be implemented by linear combination of textual and visual scores.

For future work, we aim to combine the textual information with more than just one visual descriptor. Moreover as the visual information importance depends on the query, we also plan to learn a different $\alpha$ parameter for each query.

\section{Acknowledgements}

This work was partly supported by the LIMA project ${ }^{2}$ and the Web Intelligence project $^{3}$ which are 2 Rhône-Alpes region projects of ISLE cluster ${ }^{4}$.

\section{References}

1. Halil Bisgin. Parallel clustering algorithms with application to climatology. Technical report, Informatics Institute, Istanbul Technical University, Turkey., 2008.

2. Frédéric Jurie and Bill Triggs. Creating efficient codebooks for visual recognition. In International Conference on Computer Vision, 2005.

3. David G. Lowe. Object recognition from local scale-invariant features. In Proceedings of the International Conference on Computer Vision ICCV, Corfu, pages 1150-1157, 1999.

4. K. Mikolajczyk, T. Tuytelaars, C. Schmid, A. Zisserman, J. Matas, F. Schaffalitzky, T. Kadir, and L. Van Gool. A comparison of affine region detectors. International Journal of Computer Vision, 65(1-2):43-72, 2005.

5. Christophe Moulin, Cecile Barat, Mathias Gry, Christophe Ducottet, and Christine Largeron. Ujm at imageclefwiki 2008. In ImageCLEF 2008, 2008.

6. Stephen E. Robertson, Steve Walker, Micheline Hancock-Beaulieu, Aarron Gull, and Marianna Lau. Okapi at trec-3. In Text REtrieval Conference, pages 21-30, 1994.

7. Gerard Salton, A. Wong, and C. S. Yang. A vector space model for automatic indexing. Communations of the ACM, 18(11):613-620, 1975.

8. C. Zhai. Notes on the lemur tfidf model. Technical report, Carnegie Mellon University, http://www.lemurproject.com, 2001.

\footnotetext{
${ }^{2}$ LIMA project: http://liris.cnrs.fr/lima/

${ }^{3}$ WI project: http://www .web-intelligence-rhone-alpes.org/

${ }^{4}$ ISLE cluster: http://ksup-gu.grenet.fr/isle
} 